\title{
A Discovery of the Relevance of Eastern Four-valued (Catuskoti) Logic to Define Modular Transformations When There are Multiple Ways of Representing the Same Modular Transformation
}

\author{
Madanayake R S*, Dias G K A, Kodikara N D \\ School of Computing, University of Colombo, Sri Lanka
}

Copyright (C) 2015 by authors, all rights reserved. Authors agree that this article remains permanently open access under the terms of the Creative Commons Attribution License 4.0 International License

\begin{abstract}
There were two methods of doing modular transformations from Entity Relationship Diagrams to Class Diagrams according to international researchers. In order to establish which method would best suit software engineers, we conducted a survey by giving a group of students in the computer science field, whom we considered potential future software engineers. The results we got were valid, but did not match those of any of the international researchers. We found that this situation could only be explained using Eastern Four-Valued logic, also known by such names as Catuskoti and Tetralemma.
\end{abstract}

Keywords Catuskoti, Four-valued Logic, Tetralemma, Modular Transformations, ERD to Class Diagram, Boolean Logic, Both Affirmation and Negation, Neither Affirmation nor Negation

\section{The Initial Motivation for This Experiment}

The research question for the MPhil which the first Author of this paper was involved in was "How could the different types of transformations of Software Engineering Models be combined to Expedite the Software Engineering Process?". For a solution to this question, we first tried to get a group of students to do an exercise to find out which of the two methods given in the international publications they will use to transform an ERD diagram into a class diagram.

\section{Objectives of the Survey/Experiment of the Student Assignment}

The original objective of our experimental survey was not to invalidate or undermine Aristotelian Two-Valued Logic, but to evaluate which of the methods of ERD to Class diagram (modular transformation) rules (already used in the published literature of the international researchers as given in [5] and [6]), would be used by our students for the relevant modular transformation. The conclusion which we actually wanted to arrive at was that one of the Modular Transformation Methods (out of the two suggested by the authors of [5] and by the authors of [6]), was correct, in order to use it as a Benchmark or a Basis in order to Program a software which could convert a drawn ERD Diagram into its compatible class diagram.

Since we originally theorized that the least logical method (eg. [5]) would be discarded by the students, who would choose the other Method (eg. [6]), we first classified the [6] Method as True, and the [5] Method as falls, as a sort of Hypothesis (not for a research Degree, but for the more limited purpose of publishing in a conference or in a / journal).

What happened during this experiment was actually a significant deviation from our original Objective(s), and we appreciate it if our peer reviewers could evaluate this paper in that context.

\section{Introduction}

This particular experiment is part of a main research which focuses on Modular transformations as well as on Ontologies [1], [2], [3], [4], [www1]. Modular transformations have today become an interesting research topic. One reason for this is that there are many legacy systems designed using legacy system development models, and in order to make them compatible with modern object oriented models to enable effective maintenance and modification of those systems, it has become a requirement to transform those existing legacy design models into object oriented models. Two main output diagrams obtained from 
the same modular transformation were the output diagrams obtained by two groups of international researchers (eg. Tran et.al. [5] and Fries [6], [7]) by transforming Entity Relationship Diagrams (ERDs) to Class Diagrams. To see which of these two output diagrams would be more logical, we assigned a group of computer science students whom we considered prospective software engineers, the task of doing the same modular transformation (ERD to Class Diagram) which was done by both Tran et.al. (whose was assumed as the "False" output) and Fries (whose was assumed as the "True" output) above.

To our surprise we found that most of the students who had done the modular transformation had obtained an entirely new output diagram which we also found was logically correct. To explain this surprising result, we found that it is more appropriate to use Eastern Four-Valued logic or Catuskoti [www2], [www3].

\section{Background}

Scientific reasoning in the western cultural context has been for a long time based on two valued logic, which is supposed to have arisen in Ancient Greece due to philosophers such as Aristotle.

This is also known as Boolean Logic, in relation to the two states " 1 " and "0" (comparable to the two values "true" and "false") with respect to Boolean Algebra.

According to [www4] the 3 laws of this Aristotelian Logic are as follows;

\section{The Law of Identity - A is A}

Everything is the same as itself; or a statement cannot not remain the same and change its truth value.

\section{The Law of Non-Contradiction - NOT (A and not A) - or} Nothing can both be A and Not-A

Nothing can both exist and not exist at the same time and in the same respect; or no statement is both true and false.

\section{The Law of Excluded Middle - Either (A or not A)}

Something either exists or does not exist; or every statement is either true or false.

Two of the Aristotelian laws of thought - the "The Law of the Excluded Middle" which simplistically means than everything must either be A or Not A; and the law of non-contradiction which means that nothing can both be A and Not-A [8] (which really signifies the Boolean Two valued Logic) - were (and in many respects, still are) fundamental in formal logic which is also the study of inferences and so are important in both modern science and modern mathematics [www9].

\section{Are There Drawbacks in Applying Aristotelian Logic to Experimental Results?}

However, is Boolean / Aristotelian two valued Logic really able to help in the analysis of all the different kinds of objects, phenomena and their interrelationships which exist in the world? The purpose of researching quotes of Academic Oriented personalities on the Drawbacks / Limits of Aristotelian Logic, was for us to better understand why the majority of our students arrived at an Object Diagram which was previously unforeseen by us, the Researchers who planned and carried out this experiment, initially expecting quite a different result from that which we got.

To give hypothetical example (about the limitations of Aristotelian Logic) from our own personal understanding; in the beginning of electronic computers, the two states of the vacuum tubes which represented data units inside computers were perfectly and adequately represented by Boolean Logic. Because of this, Logic gates could be designed and the used to develop computer programming logic further. However, with the development of the transistor, there was the introduction of three electronic states (Active mode, Saturation mode and Cut-off mode).

Out of these, only the Saturation mode and Cut-off mode are used for Digital computers while the Saturation mode seems to have been totally ignored (It seems to be totally outside the Boolean "Universe" of computer Machine Code.).

While this is our own personal understanding, it seems that the fact that there are limits to the capabilities of Boolean Logic has (to varying extents) been validated by the observations of other philosophers, authors and researchers.

As further examples to illustrate this point, a reading of the quotes of just three of these eminent personalities (who found it difficult to agree to a Universal Application of Aristotelian Logic) would serve to highlight this point with respect to the weaknesses of Aristotelian / Boolean two-valued logic in particular, and of Aristotelian Logic in general.

Those relevant quotes are as given below;

Quote 1:

As the author L. Zadeh stated in a publication in1975 that,

"In particular, treating Truth as a linguistic variable with values such as true, very true, completely true, not very true, untrue, etc., leads to what is called fuzzy logic. By providing a basis for approximate reasoning, that is, a mode of reasoning which is not exact nor very inexact, such logic may offer a more realistic framework for human reasoning than the traditional two-valued logic. [www6]"

The limits of Boolean Logic became especially evident in this case where another type of logic called Fuzzy Logic was needed [www6] in knowledge areas from control theory to artificial intelligence. Fuzzy Logic was mainly needed due to the weaknesses of the Law of the excluded middle, but in this particular case, it was nothing but re-including the (formerly excluded) "middle". There was no thinking out of the box, thinking of other "dimensions" of values, or going 
perpendicular to the "middle" between the two extremes.

It could be compared to allowing yourself to look at the total range of the box including its middle as well, whereas, in Boolean / Aristotelian Logic (Law of the Excluded Middle) you were firmly stuck to the sides of the box unable to either go in or out.

Quote 2:

According to Robert Maynard Pirsig an American writer and philosopher, and the author of philosophical novels, also outlines a certain different failure of the simple two-values logic in his novel "Zen and the Art of Motorcycle Maintenance".

The authors of this paper view this Quote 2 as an attempt to try to look beyond the box, (although no specific experiment was mentioned in this webpage resource from where this quote was extracted from).

He highlights it in this way with respect to a "state" referred to as "Mu" in the following way:

"For example, it's stated over and over again that computer circuits exhibit only two states, a voltage for "one" and a voltage for "zero." That's silly!

Any computer-electronics technician knows otherwise. Try to find a voltage representing one or zero when the power is off!

The circuits are in a mu state." [www7]

Quote 3:

Dr. Nalin De Silva, (who is a retired Professor and was the former Dean of the Faculty of Science Kelaniya / Vidyalankara University of Sri Lanka) who has a $\mathrm{PhD}$ in Theoretical Cosmology (University of Sussex, UK.) had this to say regarding the failure of Aristotelian / Boolean Logic:

"However, the logic that is abstracted from Aristotelian-Newtonian Experiences is not capable of dealing with change in general and motion in particular. It is demonstrated by the famous Zeno's paradox that deals with an arrow in motion. The Aristotelian logic is faced with contradictions when it is employed to describe motion and one would end up by showing that motion is impossible! The Calculus of Newton and Leibniz, though their approaches were not the same, tried to get over this difficulty using infinitesimals intuitively without formally defining them. However, infinitesimals were not liked by the western Mathematicians and Philosophers and there were objections to these "ghosts" by people such as Berkeley. Euler, one of the greatest western Mathematicians with an intuition that surpassed most of the others freely used infinitesimals in his formulation of Mathematical Analysis. However, as the western Mathematicians did not like these infinitesimals that according to Berkeley were neither finite nor not finite, later Mathematicians Dedekind, Cantor and Cauchy "exorcised" infinitesimals from Mathematical Analysis and introduced what is known as the epsilon - delta definition of limit, which is based on Aristotelian logic. The calculus that tried to deviate from Aristotelian logic at the beginning was brought back to an "arithmetical" definition based on that logic in the nineteenth century. It is interesting to note that something similar is happening in Quantum Physics. Bohr (and Heisenberg) who tried to deviate from the Classical Physics world view in the thirties created what is known as the Copenhagen Interpretation of Quantum Physics. Copenhagen Interpretation was obviously not in agreement with Aristotelian logic and the tendency at present is to formulate a new interpretation based on Aristotelian logic and doing away with Heisenberg's uncertainty Principle." [www8]

\section{Methodology}

Originally, the purpose of our research was not to find any incompatibilities with Aristotelian / Boolean Logic, but to see whether the Method used by Tran et.al. or the Method used by Fries would be more feasible or more realistic for doing Modular Transformations from ERDs to Class Diagrams (or in other to see words which of the two considered methods would be more appropriate to transform an ERD diagram to a Class diagram).

To achieve the above, what we decided to find out was, whether a group of prospective future software engineers (eg.: computer science degree students) would use Tran's Method or Fries' Method to do the Modular Transformation from ERD to Class Diagram and to see whether all students would follow the same method or else, if some students would select Tran's Method and if others would select Fries' Method to do their assignment, to reach a conclusion based on which method would be followed by the majority of the students.

The following was the input diagram or the source diagram which was the Legacy ERD diagram that was given to the students to convert into a Class diagram (Figure 01). This source for the case study was obtained by us from the relevant research paper by Fries [6], while Fries had apparently got it from a literature attributed to Yourdon known as "Modern Structured Analysis" [9]. 


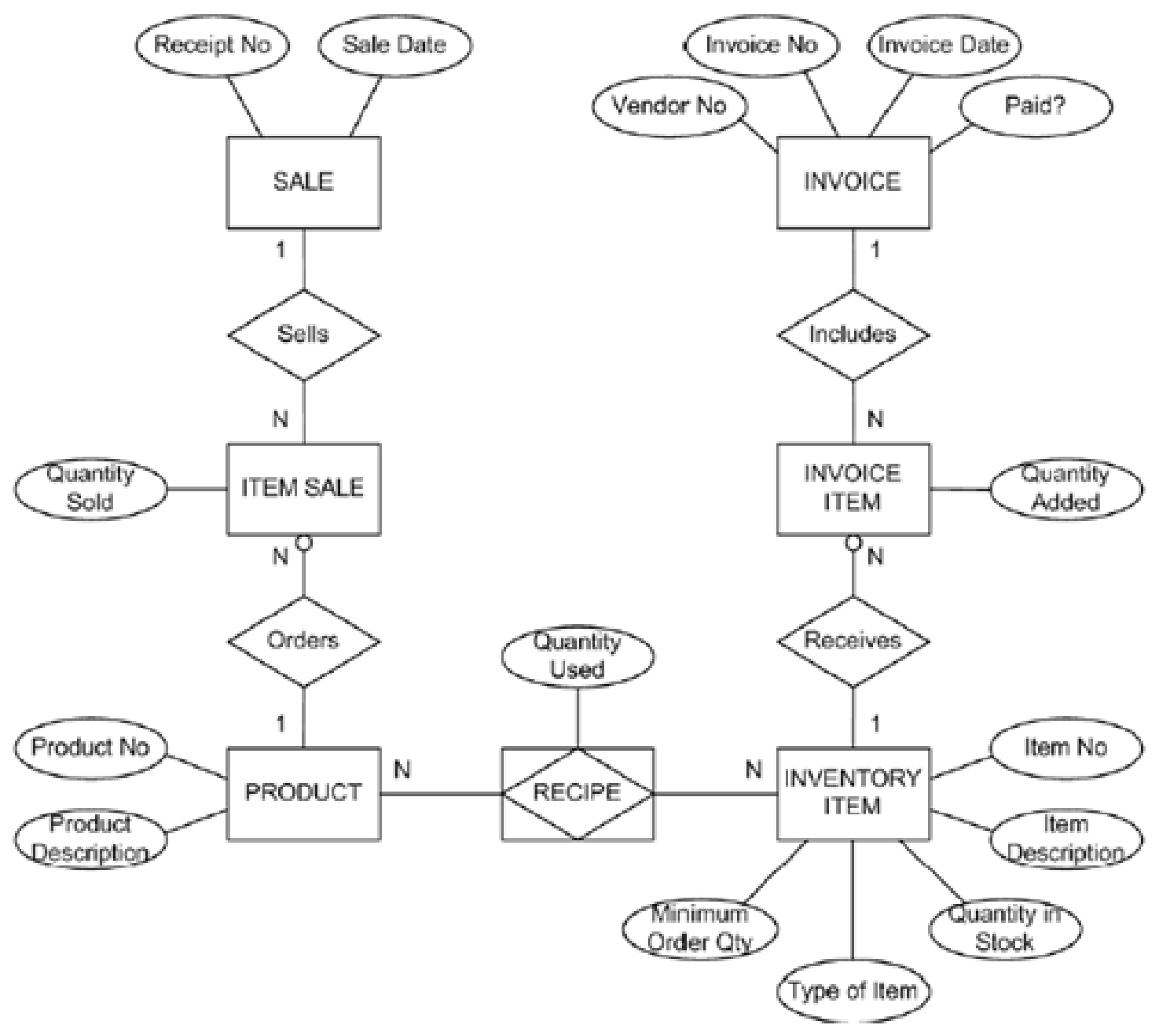

Figure 1. The Input / Source ERD Diagram given to the students to be transformed into a Class Diagram [6], [9]

The relevant experiment was conducted as an assignment where a batch of students of the Science Faculty of the University of Colombo were required to transform the given ERD diagram (Figure 01) encountered in Structured Systems Analysis and Design (SSADM) which is a Legacy Design Methodology, into a Class diagram found in Unified Modelling Language (UML).

Please NOTE that the following output diagrams are ALL from the published research papers of Tran and Fries [5], [6]. This is the reason that figure 3 appears to be Different from figures 2 and 4, since figure 3 is from Tran [5], figure 2 is from Fries [6], and figure 4 is just a Modified version of figure 2, which was done to give a generalized form of the common Output diagram produced by the majority (or 171) of our students.

There are two major output Class diagrams when transforming an ERD diagram to a Class diagram using the ERD of the Hoosier-Burger case study as the Input diagram, that of Fries (Figure 02) and that of Tran et. al (Figure 02).

Our expectation was that the students would transform the input diagram into the output diagram produced by Fries, which we considered as the True / Yes Boolean value - since Fries had already evaluated Trans' paper which can before and Fries seemed to us to have "corrected" some of the rules used previously by Tran [5], such as disposing of the Transformation of Relationships between ERD Entities into Methods in the compatible Class Diagram (which was one of the rules followed by Tran), while the output diagram produced by Tran we considered as the False / No Boolean value since Fries seemed to have already studied Trans' paper, an declared some of the Transformation Rules used by Tran to be "Invalid".

The assignment was given as part of their subject module called Visual Programming Technologies subject as $50 \%$ of the marks for the project (which maps to $10 \%$ of the marks for the entire Visual Programming Technologies subject module).

They were not taught about either the Tran's or the Fries' transformation methods in order to prevent experimental bias.

Please be kind enough to NOTE that although we the researchers have been researching publications dealing with Modular Transformations from ERDs to Class Diagrams since the beginning of the Year 2011 (for well over Four Years!) we could still only find the two Modular Transformation Methods (from ERDs to Class Diagrams) 
used by Tran and by Fries, in the International Research Arena.

So, to date, we have found only the Algorithmic Methods used by Tran and by Fries, in the International Research Arena, for this particular Modular Transformation.

If there are Three or More other methods to do the Modular Transformations from ERDs to Class Diagrams, then we Obviously have NOT found them, Which is Very Unfortunate (as well as highly improbable) from the point of view of this research paper.

The output Class Diagram generated using the Fries' Method is shown below (Figure 02).

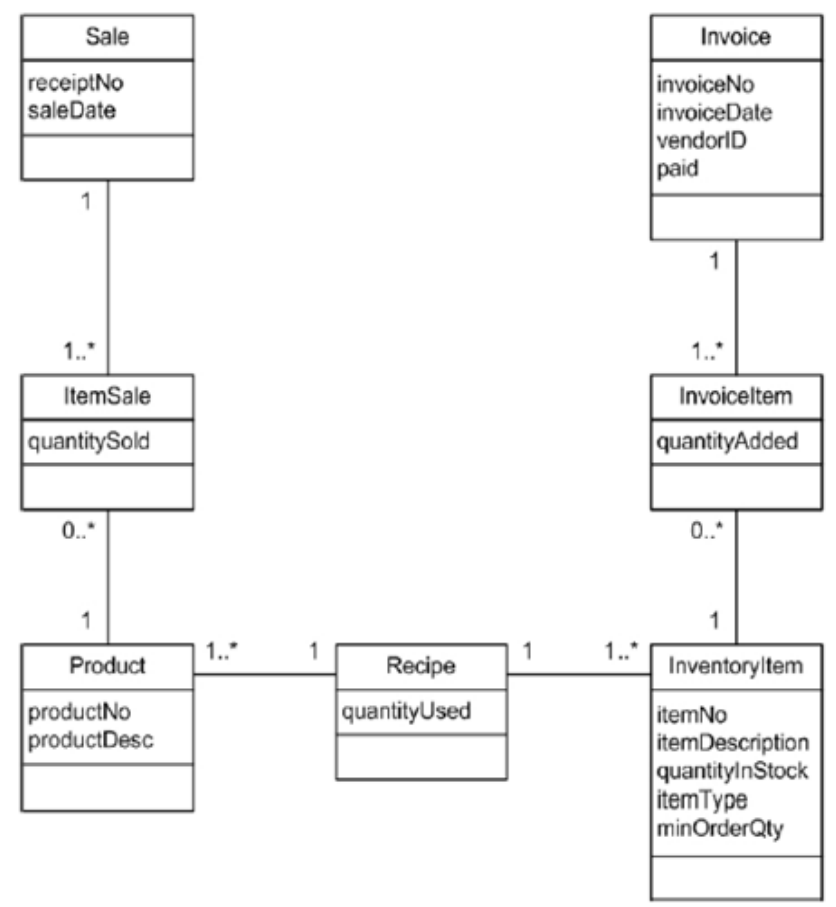

Figure 2. The Output Class Diagram Generated by the Method followed by Fries, T. P. given here as Fries' Method [6]

The output Class Diagram generated using the Tran et.al.s' method is shown below (Figure 03).

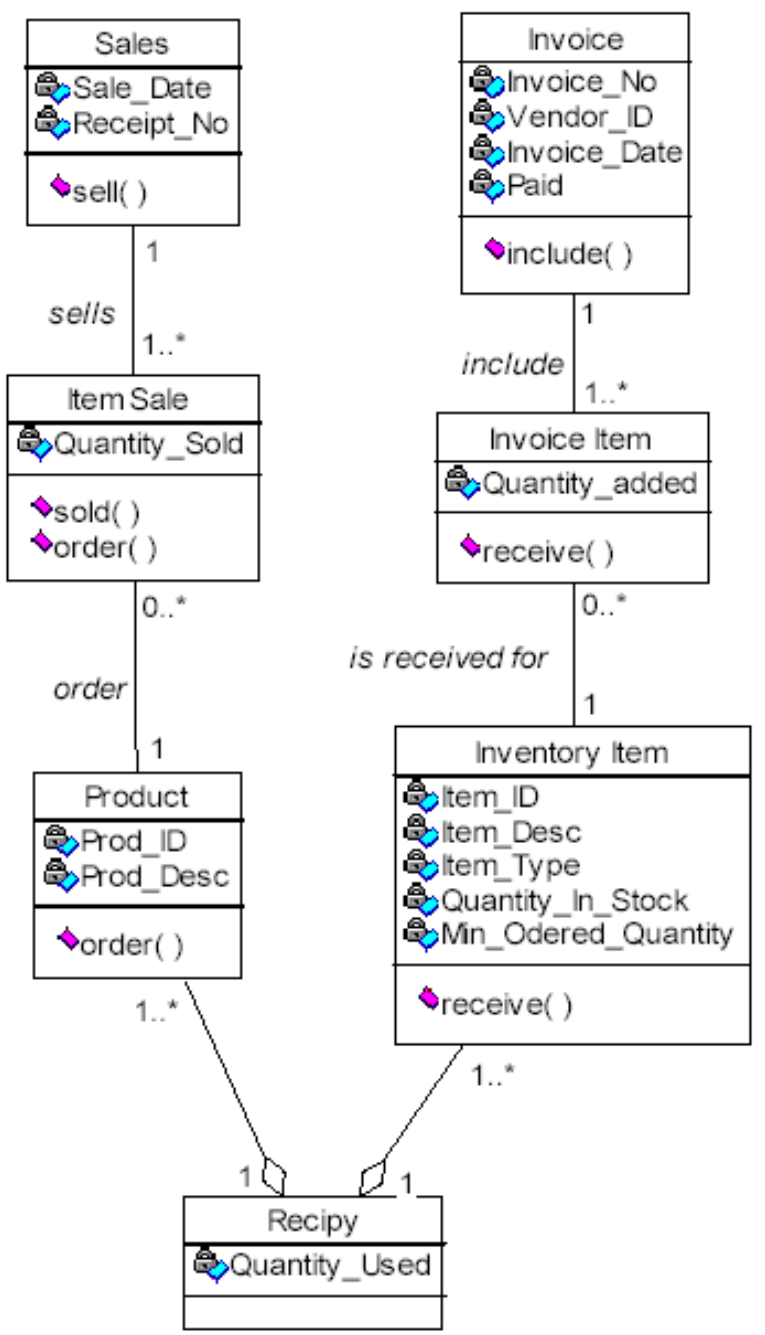

Figure 3. The Output Class Diagram Generated by the Method followed by Tran, T.N., Khan, K.M., Lan, Y.C. given here as Tran's Method or Tran et. al.'s Method [5]

An example of the output achieved by the majority of students is shown below (Figure 04) which could have been achieved Neither by following Fries' Method Nor by following Tran's Method. 


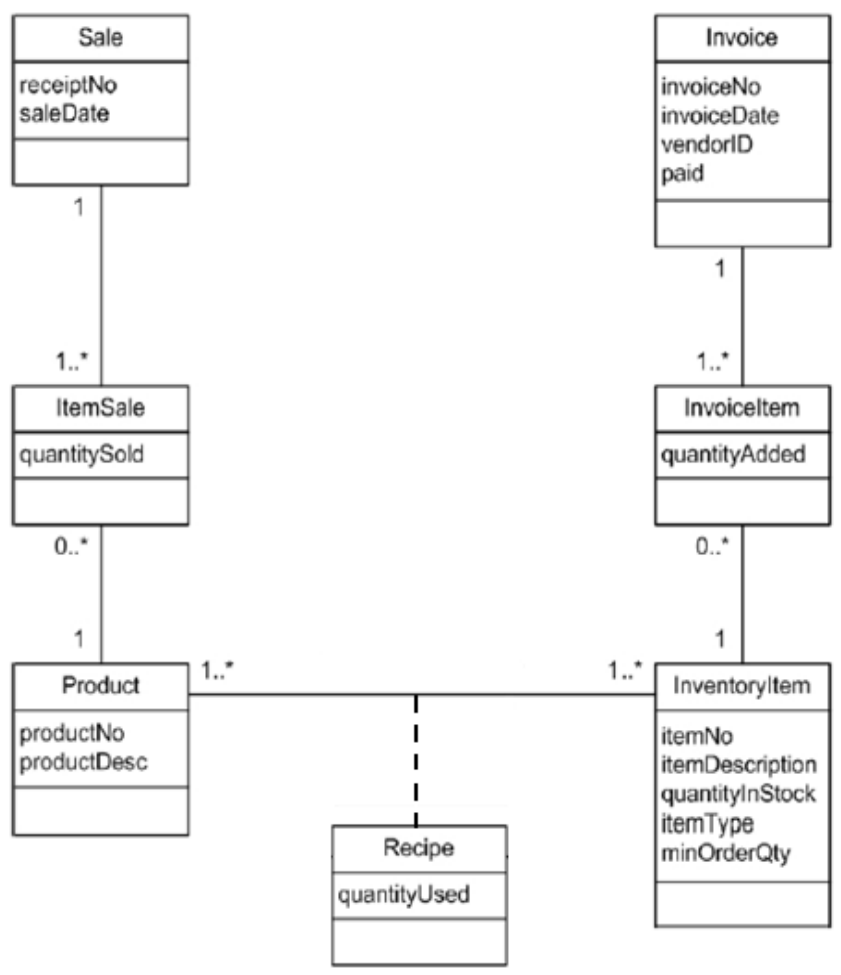

Figure 4. An Example of the Output Class Diagram Generated by 171 of the Students

The actual Method, and / or the rules of the Transformation which these students used, could not be extracted from the students at this point (since they were not asked or taught to follow any rules or a Method in this particular Modular Transformation), which should be looked at as a future work in this ongoing Research Project.

Please note that it is the final Output Diagram generated by 171 of the students which we considered as most important for this particular Research Paper rather than the exact Rules of the Method which the students followed.

Please also note that the "Recipe" class at the bottom of the above diagram (Figure 04) is actually out of the main Diagram Structure, and only connects to the main Structure by a Dashed Line (signifying the case of a "Weak" Relationship between the other six classes). This is certainly not the case in Either the output generated by Fries' Method (Figure 02) Or that generated by Tran's Method (Figure 03).

In other words, the students seemed to have generated a totally Unique output which Neither Fries' Nor by Tran's research groups could even seem to be able to visualize - a totally out of the box solution for a particular case study within this limited domain of ERD to Class Diagram Modular Transformations.

\section{Results}

We got the following results from this experiment with regard to the number of assignments which showed which method was followed.

1) Fries' Method $=06$ assignments

2) Trans et. al.'s Method $=00$ assignments

3) Both Methods (Hybrid) $=01$ assignment

4) Neither Method = 176 assignments

5) Neither Method (But Specific 3rd Method) $=171$ assignments

From the results it could be seen that many students (176 of them) got neither output diagram as their result. Although 5 of those transformations were wrong since some of the entities in the ERD were not represented as classes in the relevant Class diagram, making the number of classes less than the number of respective entities, we filtered them out, but still we had 171 assignments that were correct and had received a specific result which neither followed Trans' nor Fries' method.

It is also significant that at least one student achieved a "Hybrid" result leading one to come to the conclusion that both Trans' and Fries' methods could be merged to give a result on some occasions (specifically depending on the practical system under study). In this particular case the number of classes (7 classes) was not violated.

The results (once we filtered out the results which we considered wrong), are given in the table (Table 01) below.

Table 1. Representation of the final student assignment outputs which the Authors considered as correct according to the UML rules

\begin{tabular}{|c|c|}
\hline $\begin{array}{c}\text { Method followed by the student(s) to } \\
\text { convert an ERD Diagram into a Class } \\
\text { Diagram }\end{array}$ & $\begin{array}{c}\text { Number of Assignment } \\
\text { Outputs in each case }\end{array}$ \\
\hline Fries' Method & 6 \\
\hline Trans et. al.'s Method & 0 \\
\hline Both Methods (Hybrid) & 171 \\
\hline $\begin{array}{c}\text { Neither Method (But Specific 3rd } \\
\text { Method) }\end{array}$ & \\
\hline & \\
\hline
\end{tabular}

In the Chart given on the next page, The first (left most) column maps to the Yes/True Boolean value, and the middle left column on this Chart maps to the No/False Boolean value (But does NOT have any data since none of the students generated that Output Diagram). The two Columns to the right Do Not Map to any of the Boolean Values, and seem to be Outside of Aristotelian / Boolean Logic with respect to this particular experiment.

Kindly go through the chart on the next page (page 12). 


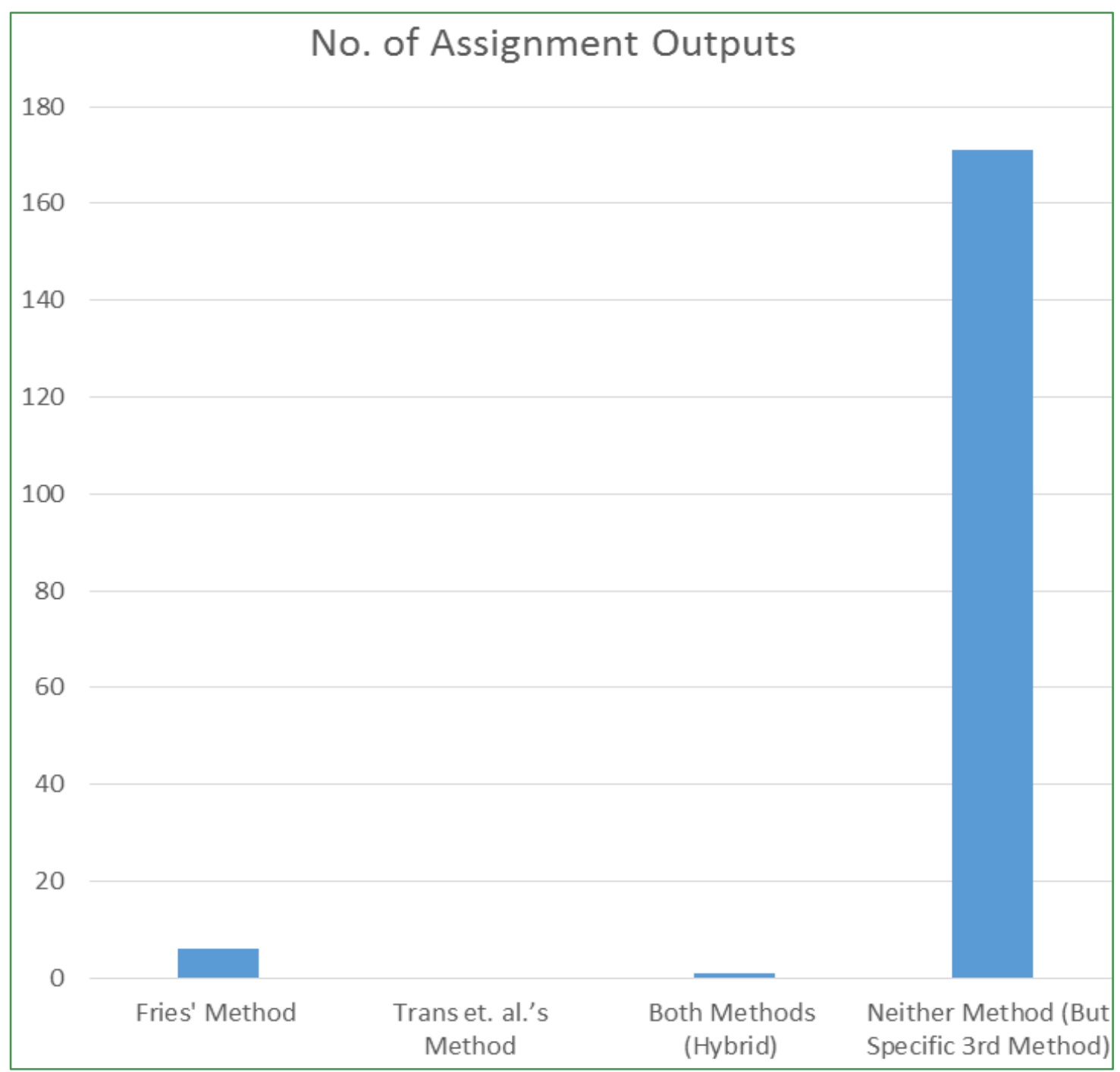

(The Columns Extreme Left "True", Middle Left "False", Middle Right "Both True AND False", Extreme Right "Neither True Nor False")

Chart 1. The same results in Table 01 are given in a more graphic

\section{Results \& Discussion}

From this data it can be seen that the large majority of students got a specific unique output that was generated by Neither Tran Nor Fries who were the international researchers (research groups), whose work we considered as the basis for this particular experiment.

In our domain of transformation of ERDs to Class Diagrams, we considered the output diagrams of Tran and Fries as the only two correct possibilities. Since Fries was the later researcher who had also criticized Trans' work, we identified Fries' output as True (affirmation) and Trans' output as False (negation).

However, the existence of a $3^{\text {rd }}$ possibility which was compatible with neither the outputs of Tran nor the outputs of Fries was a totally unexpected and surprising result.

Generally a proposition in Boolean / Aristotelian or Classical logic or Two Valued Logic (which is of Western origin) is that there are only two possible values - affirmation (true or X) or negation (false or $\neg \mathrm{X}$ ) leading to conclude that only the methods of Fries and that of Tran were possible in the case of this particular experiment.

While analyzing our unique result, we came to the conclusion that the simple true / false logic (where affirmation or negation were the only two possibilities) was not enough to make sense of the Total Output of this particular experiment.

We found it more appropriate to use Eastern (Catuskoti or Tetralemma) logic [www2] where a proposition could have four values as possible answers.

Those 4 possible values found in Tetralemma logic (Catuskoti or Four Valued Logic) are as shown below [www2];

$\mathrm{X}=$ affirmation (True)

$\neg \mathrm{X}=$ negation (False)

$\mathbf{X} \wedge \neg \mathbf{X}=$ both affirmation and negation (Both True And False)

$\neg(\mathbf{X} \vee \neg \mathbf{X})=$ neither affirmation nor negation (Neither True Nor False)

The numbers of students who followed the different 
methods to do the assignment (as given in the Results section above) are shown against the appropriate values of Tetralemma logic in the Table below (Table 02).

Table 2. The results obtained by us are shown against the relevant values of Tetralemma logic in this table

\begin{tabular}{|c|c|c|c|}
\hline $\begin{array}{c}\text { Tetralemma } \\
\text { Logical } \\
\text { Value } \\
\text { (Symbolic) }\end{array}$ & $\begin{array}{c}\text { Meaning of } \\
\text { the Logical } \\
\text { Value }\end{array}$ & $\begin{array}{c}\text { Relevant Practical/ } \\
\text { Experimental } \\
\text { Situation (The } \\
\text { method followed to } \\
\text { do the assignment) }\end{array}$ & $\begin{array}{c}\text { Number of } \\
\text { Relevant } \\
\text { Assignment } \\
\text { outputs in each } \\
\text { case }\end{array}$ \\
\hline $\mathrm{X}$ & affirmation & Fries' Method & 6 \\
\hline$\neg \mathrm{X}$ & negation & $\begin{array}{c}\text { Trans et. al.'s } \\
\text { Method }\end{array}$ & 0 \\
\hline $\mathrm{X} \wedge \neg$ & $\begin{array}{c}\text { both } \\
\text { affirmation } \\
\text { and negation }\end{array}$ & $\begin{array}{c}\text { Both Methods } \\
\text { (Hybrid Method) }\end{array}$ & 1 \\
\hline$\neg(\mathrm{X} \vee$ & $\begin{array}{c}\text { neither } \\
\text { affirmation } \\
\text { nor negation }\end{array}$ & $\begin{array}{c}\text { Neither Method } \\
\text { (But Specific 3rd } \\
\text { Method) }\end{array}$ & 171 \\
\hline
\end{tabular}

In our situation, 176 of the outputs (specifically the 171 outputs of those which we identified as correct), followed neither the Fries' method nor the Trans' method. Therefore it corresponds to the $\neg(\mathrm{X} \vee \neg \mathrm{X})$ (neither) affirmation nor negation possibility found in Tetralemma logic (Refer Table 02, above).

As a reason for the fact that none of our students used Tran's method (since none of them failed to get that output) we hypothesize the lack of consideration by the students that the relationships in an ERD Diagram could be transformed into the methods in a class diagram could have prevented students from even considering that such a method was possible; specially since the Methods of ERD to Class diagram conversion was not taught to the students before the conversion. However, at the current moment, this remains purely hypothetical since the curriculum did not provide enough time schedule to interview the students and find out the reasons.

\section{Conclusions and Future Work}

According to the results of this experiment, the authors / researchers could conclude that at least in this experiment of this type, the use of Aristotelian two-fold logic (true / false) maybe inadequate to evaluate the validity of a hypotheses.

If the Eastern four-fold logic was applied, a result more compatible to reality could be obtained. In that case, a hypotheses could be True, False, Both True and false; or, neither True nor false.

As future work, one could also further analyze the results of the following as given in the results section above.

- Neither Method = 176 assignments

- $\quad$ Neither Method (But Specific 3rd Method) $=171$ assignments

Although 5 of the assignments in "4)" were identified as incorrect, leaving us with only 171 assignments (since $176-$ $5=171$ ), as shown in "5)", we could only come to this correct / incorrect assumption due to the data provided to us by the other cited authors as given in [5] and [6]. As to whether these 5 assignments are absolutely incorrect has to be verified by re-examining the actual business environment of the "Hoosier-Burger" case study from which the data for the original ERD diagram was extracted. If future authors could find this situation today, they could check the validity of the incorrect assignments. However, the authors wish to state that this Anomaly does not have any bearing on the conclusions regarding the requirement of Eastern four-fold logic, since our input data did not rely on the actual business environment of the "Hoosier-Burger" case study, but on testing the feasibility of transforming an already created Entity Relationship Diagram (ERD) into a Class Diagram.

Another possible future work would be to find out the exact rules and / or logic the students used to convert generate the unique output class diagram that 171 of them generated (as shown in Figure 04 of page 9, above).

Finally, A Comparison of Tetralemma Logic with Fuzzy Logic to further Highlight the significance of the Conclusions arrived at in this Research

We wish to give the readers a brief comparison of Tetralemma with fuzzy logic to help justify the fact that Tetralemma logic is more applicable in the case of the results of this experiment than Fuzzy Logic.

Fuzzy Logic deals more in bridging the gap between the two extremes of True and False, and has more to do with dealing with the restrictions of the Law of the Excluded middle. However (fuzzy logic) still remains within the confines of the two extremes of Logic, or within or limited by) the range.

Tetralemma Logic on the other hand forces one to think beyond the confines of two extremes within the same range of values. Tetralemma Logic deals wiith the un-thought, unanticipated, unexpected "other-dimensional" type realities than what one could assume would be the possible range of values (for a particular variable), before an experiment is done. Tetralemma forces one to think of the possibility of other ranges of values or dimension which the researchers had not thought before doing the experiment.

In this case we conclude that Tetralemma is more applicable, since the unexpected results we received were definitely not ones which we could place either on one of the extremes, or between the two extremes.

\section{REFERENCES}

Web References

[www1]

http://en.wikipedia.org/wiki/Ontology_(information_scienc

e) Last accessed 06-05-15

[www2] http://en.wikipedia.org/wiki/Tetralemma Last accessed 06-05-15

[www3] http://en.wikipedia.org/wiki/Catușkoṭi Last 
accessed 06-05-15

[www4]

http://oregonstate.edu/instruct/phl201/modules/Philosophers

/Aristotle/aristotle_laws_of thought.html

Last accessed 16-10-15

[www5]

https://en.wikipedia.org/wiki/Mathematical_logic\#19th_cen tury

Last accessed 14-10-15

[www6]

http://www.sciencedirect.com/science/article/pii/002002557 5900365

Last accessed 11-10-15

[www7] https://en.wikipedia.org/wiki/Mu_(negative)

Last accessed 11-10-15

[www8] http://www.island.lk/2004/05/05/midwee03.html

Last accessed 11-10-15

[www9]

https://en.wikipedia.org/wiki/Logic\#The_study_of_logic 14-10-15

[1] Tilakaratna, P., Rajapakse, J. (2011), Forward Engineering the Object Oriented Analysis and Design, 107-112., Proceedings of the 5th Malaysian Conference in Software Engineering.

[2] Benevides, A.B. and Guizzardi, G.,(2009) A Model Based Tool for Conceptual Modeling and Domain Ontology Engineering in OntoUML, (Lecture Notes in Business Information Processing, vol. 24), p. 528-538. ISBN
978-3-642-01346-1.

[3] Tilakaratna P., and Rajapakse J., (2012) "Ontological Framework for Object-Oriented Analysis and Design," 251-260. In American Journal of Engineering and Applied Sciences 5 (3).

[4] Li X., and Parsons J., (2007) "Ontological Semantics for the Use of UML in Conceptual Modeling," In: Proceedings of Tutorials, posters, panels and industrial contributions at the 26th International Conference on Conceptual Modeling - ER 2007 Auckland, New Zealand. CRPIT, 83. Grundy, J., Hartmann, S., Laender, A. H. F., Maciaszek, L. and Roddick, J. F., Eds. ACS. 179-184.

[5] Tran, T.N., Khan, K.M., Lan, Y.C. (2004), A framework for transforming artifacts from Data Flow Diagrams to UML. In: Proceedings of the 2004 IASTED International Conference on Software Engineering, Innsbruck, Austria.

[6] Fries, T.P. (2006), A Framework for Transforming Structured Analysis and Design Artifacts to UML., Proceeding of the $24^{\text {th }}$ annual conference on Design of communication, USA.

[7] Jilani A.A.A., Usman M., Nadeem A., Malik Z.I. and Halim Z., (2011), "Comparative Study on DFD to UML Diagrams Transformations," World of Computer Science and Information Technology Journal (WCSIT), vol. 1, pp. 10-16.

[8] Kodish, B.I, "Dare to Inquire: Sanity and Survival for the 21st Century and Beyond", pp 166.

[9] Yourdon, E., Modern Structured Analysis. Prentice Hall (India) Pvt. Ltd., 1996. 